\title{
Mixed Lubrication Simulation of Hydrostatic Spherical Bearings for Hydraulic Piston Pumps and Motors
}

\author{
Toshiharu KAZAMA** \\ ${ }^{* *}$ Department of Mechanical Systems Engineering, Muroran Institute of Technology, \\ 27-1, Mizumoto-cho, Muroran, Hokkaido, 050-8585, Japan \\ E-mail: kazama@mmm.muroran-it.ac.jp
}

\begin{abstract}
Mixed and fluid film lubrication characteristics of hydrostatic spherical bearings for swash-plate-type axial piston pumps and motors are studied theoretically under non-steady-state conditions. The basic equations incorporating interference and contact of surface roughness are derived fundamentally through combination of the GW and PC models. Furthermore, a programming code that is applicable to the caulked-socket-type and open-socket-type bearings is developed. Effects of caulking, operating conditions, and the bearing dimension on the motion of the sphere and tribological performance of the bearings are examined. Salient conclusions are the following: The sphere's eccentricity increases in the low supply pressure period. The time-lag of the load change engenders greater motion of the sphere. Caulking of the bearing socket suppresses the sphere's motion. The bearing stiffness increases and power loss decreases for smaller recess angles. Minimum power loss is given under the condition that the bearing socket radius nearly equals the equivalent load radius.
\end{abstract}

Key words: Tribology, Fluid Power Systems, Hydrostatic Bearings, Mixed Lubrication, Sphere, Ball Joint, Caulking, Hydraulic Piston Pumps and Motors, Simulation

\section{Introduction}

Spherical bearings can support axial and radial loads simultaneously in limited spaces of hydraulic equipment and industrial machinery. In swash-plate-type axial piston pumps and motors, a spherical bearing ${ }^{(1)}$ is mounted in a piston assembly to be used as a ball joint connecting the piston and the slipper ${ }^{(2)}$, where the pistons are the prime component of the pumps and motors. The motion and lubrication of the pistons as well as those of the slippers are influenced by the behavior of the spherical bearings. Therefore, a need exists to examine their tribological characteristics and to develop a simulation model of them.

Fundamental characteristics of both hydrostatic bearings and spherical bearings for machine elements ${ }^{(3,4)}$ have been investigated for many years and published in several journals and books ${ }^{(5)}$. The basic equations and the design charts can be referred in handbooks ${ }^{(6)}$. Even so, the operating conditions are often limited to those of full fluid film lubrication and constant supply pressure.

In fact, the spherical bearings used in hydraulic pumps and motors are rarely operated in a full film lubricating condition because the sphere diameter is smaller than the piston diameter, based on the design concept of compactness of the components: hydrostatic pressure is supplied from the cylinder bore to the spherical bearings through a restrictor; the 
bearing load is given by product of the cylinder-bore's pressure and the cross-sectional area of the pistons. Consequently, the hydrostatic load-carrying capacity is smaller than the load, and the asperities of the surfaces in contact must support a fraction of the load. In other words, the bearings are imposed to operate in mixed lubrication ${ }^{(7)}$. A discrepancy of assumptions exists between the ideal condition of fluid film lubrication and the real-world operations, which involve mixed lubrication.

A few theoretical and experimental studies of spherical bearings that are applicable to hydraulic pumps and motors have been reported. A pioneering study of this subject was contributed by Yamaguchi ${ }^{(1)}$. He applied the Reynolds equation to the hydrostatic spherical bearings while focusing on hydraulic piston pumps and motors, considering the dynamic motion of the bearings, and performed unsteady calculations in fluid film lubrication. Hazaki and Imai ${ }^{(8)}$ experimented on the hydraulic motor and elucidated the behavior of hydraulic ball pistons. They described the ball piston mechanism, but did not address mixed-lubrication problems. Fang and Ikeya ${ }^{(9)}$ performed an analytical study on the ball joint of a hydraulic motor. However, they discussed only a situation of fluid film lubrication. Recently, Yacout et al. ${ }^{(10)}$ published theoretical results of hydrostatic spherical bearings in the steady state and discussed optimum conditions including roughness effects; they did not consider asperities contacting. Therefore, no study has been done with results that apply directly to design of ball-joints with functioning hydrostatic spherical bearings in hydraulic piston pumps and motors.

The author has derived analytical solutions of hydrostatic spherical bearings under a steady state condition ${ }^{(11)}$ and has shown the optimum size based on minimum power loss and maximum stiffness for fitted-type and clearance-type bearings as well as capillary and orifice restrictors. Afterward, he constructed a theoretical model of spherical bearings in mixed lubrication, calculated tribological characteristics in a steady state, and confirmed the numerical solutions using experimental data ${ }^{(12)}$. Furthermore, he expanded the theoretical model and the programming code to an unsteady state and performed a computer simulation in unsteady conditions ${ }^{(13)}$ for non-caulked socket (open socket)-type spherical bearings.

This paper presents a dynamic simulation of hydrostatic spherical bearings applicable to ball joints of hydraulic piston pumps and motors. The bearing motion and tribological characteristics in mixed to fluid film lubrication are clarified, including the effects of caulking of the bearing socket, roughness interaction, and asperity contact.

\section{Nomenclature}

$\begin{array}{ll}C & : \text { radial clearance }=R_{2}-R_{1} \\ \bar{h} & : \text { clearance }=h / C \\ K & : \text { bulk modulus of fluid } \\ \bar{L} & : \text { power loss }=L /\left(\omega p_{s 0} R_{1}^{3} S o\right) \\ \bar{m} & : \text { mass }=\omega^{2} C m /\left(p_{s 0} R_{1}^{2} S o\right) \\ \bar{p} & : \text { pressure }=p /\left(p_{s 0} S o\right) \\ \bar{p}_{r} & : \text { recess pressure }=p_{r} /\left(p_{s 0} S o\right) \\ \bar{p}_{s} & : \text { supply pressure }=p_{s} /\left(p_{s 0} S o\right) \\ \bar{Q} & : \text { flow rate }=Q /\left(\omega R_{1}^{3}\right) \\ R_{\mathrm{w}} & : \text { equivalent load radius }=\left[W \mid /\left(\pi p_{s}\right)\right]^{1 / 2} \\ R_{1} & : \text { sphere radius } \\ R_{2} & : \text { bearing socket radius } \\ \bar{r}, \theta, \bar{z} & : \text { coordinates }=r / R_{1}, \theta, z / C \\ S o & : \text { parameter }=6 \mu \omega\left(R_{1} / C\right)^{2} / p_{s 0} \\ \bar{T} & : \text { torque }=T /\left(p_{s 0} R_{1}^{3} S o\right) \\ \bar{V} & : \text { recess volume }=p_{s 0} S o V_{r} /\left(K R_{1}^{3}\right)\end{array}$




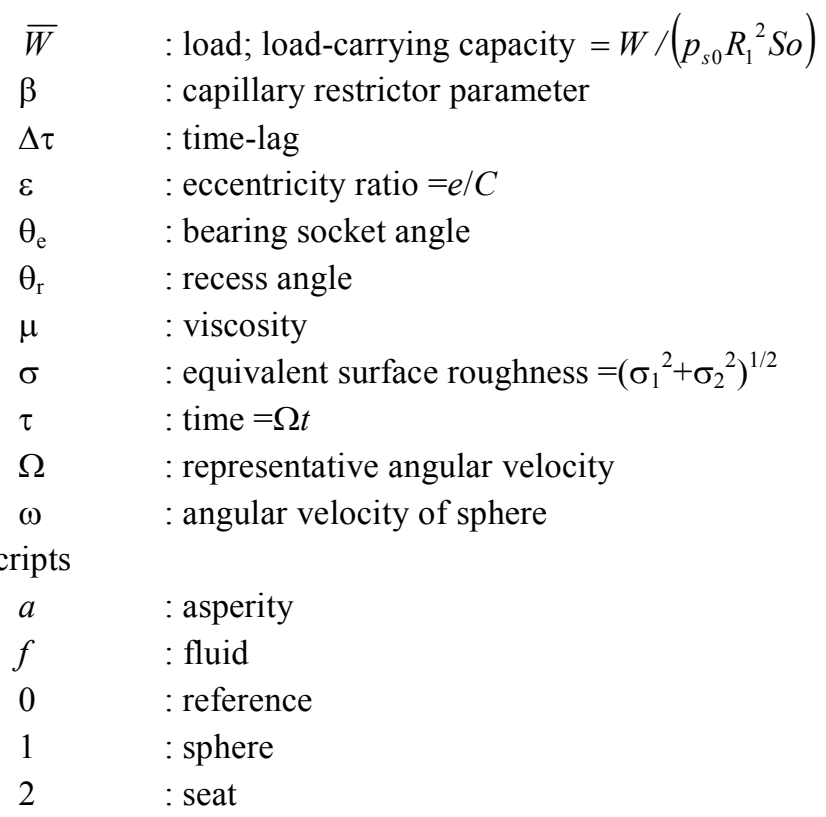

\section{Basic equations}

A typical piston assembly, which consists of a piston, a ball joint and a slipper, is shown in Fig. 1. It is the primary tribological component of swash-plate-type axial piston pumps and motors. The ball joint is the most important element, connecting the piston and the slipper; it functions as a hydrostatic spherical bearing. A schematic showing hydrostatic spherical bearings is portrayed in Fig. 2. The assumptions used for this study are: the sphere rotates around the $z$ axis at a constant angular velocity $\omega$ under the dynamic supply pressure $p_{\mathrm{s}}$ and the concentric load $W$ shown in Fig. 3, where $0<\tau<\pi / 2$ and $3 \pi / 2<\tau<2 \pi$ correspond to the high-pressure period and $\pi / 2<\tau<3 \pi / 2$ corresponds to the low-pressure one. The mixed lubrication model ${ }^{(14)}$ used for this study consists fundamentally of the asperity contact mechanism proposed by Greenwood and Williamson ${ }^{(15)}$ and the average flow model by Patir and Cheng ${ }^{(16)}$, further including the adsorbed film around asperities. The roughness is assumed as isotropic, the standard plane is rigid, and the liquid's physical properties are constant.

Following the GW model, pressure $\bar{p}_{a}$ in contact at the infinitesimal apparent contact area is given as $\bar{p}_{a}=\bar{p}_{a e}+\bar{p}_{a p}$ (subscripts $e$ and $p$ respectively denote elastic and plastic deformation of the asperity in contact), and

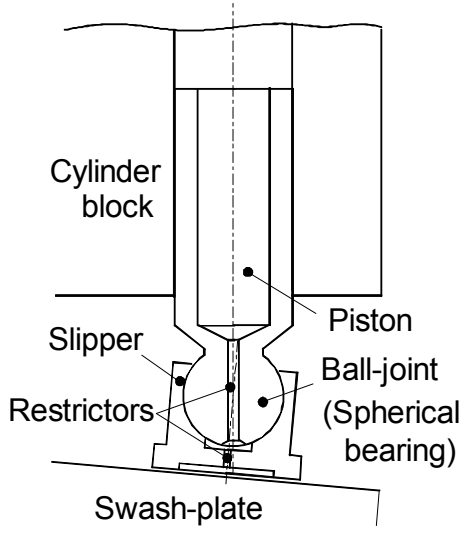

Fig. 1 Piston assembly of swash-plate-type axial piston pumps and motors 


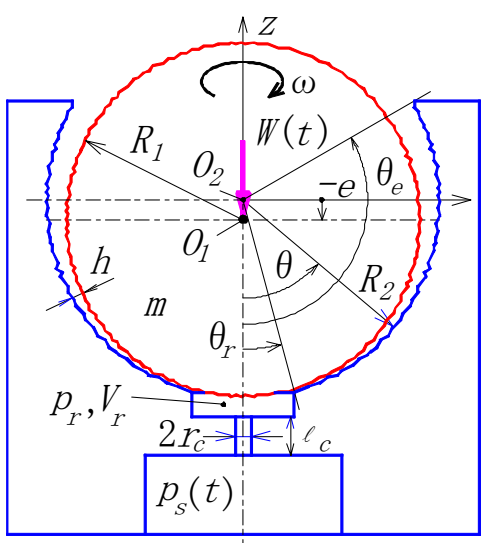

Fig. 2 Modeling and coordinates of hydrostatic spherical bearings with a caulked socket

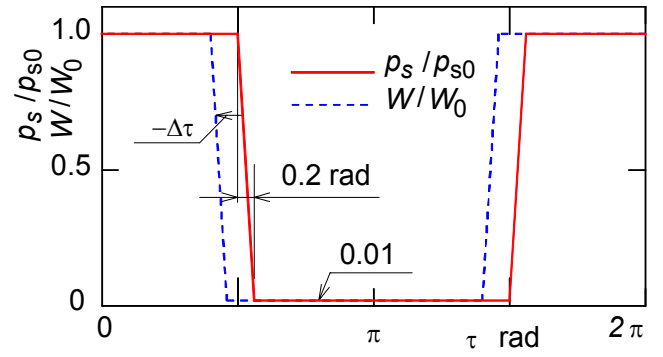

Fig. 3 Changes in supply pressure $p_{\mathrm{s}} / p_{\mathrm{s} 0}$ and load $W / W_{0}$

$$
\begin{aligned}
& \bar{p}_{a e}=\left(\frac{2}{3}\right) \bar{E}^{\prime} \eta \beta^{\prime} \sigma^{*}\left(\frac{\sigma^{*}}{\beta^{\prime}}\right)^{1 / 2}\left[F_{3 / 2}\left(d_{e}^{*}\right)-F_{3 / 2}\left(d_{e}^{*}+w_{p}{ }^{*}\right)\right] \\
& \bar{p}_{a p}=\pi \bar{H}_{a} \eta \beta^{\prime} \sigma^{*} F_{1}\left(d_{e}^{*}+w_{p}{ }^{*}\right) \\
& F_{m}\left(d_{e}^{*}\right)=\int_{d_{e}^{*}}^{\infty}\left(s-d_{e}^{*}\right)^{n} \psi(s) d s
\end{aligned}
$$

The parameters are the following: $d_{\mathrm{e}}{ }^{*}$ is separation; $\bar{E}^{\prime}$ is the equivalent elastic modulus $=E^{\prime} /\left(p_{s 0} S o\right) ; \bar{H} a$ is the hardness of softer materials $=H a /\left(p_{s 0} S o\right)$; $w_{p}{ }^{*}=\left(\beta^{\prime} / \sigma^{*}\right)\left(2 H a / E^{\prime}\right)^{2} ; \beta^{\prime}$ is the equivalent radius of asperity summit; $\eta$ is the asperity density; $\sigma^{*}=0.7 \sigma, \psi(\mathrm{s})$ is the standard probability density function deviation $=(2 \pi)^{-1 / 2} \exp \left(-s^{2} / 2\right)$.

Following the PC model, the unsteady axi-symmetric Reynolds equation is given as

$$
\begin{aligned}
& \frac{1}{\sin \theta} \frac{\partial}{\partial \theta}\left(\phi \bar{h}^{3} \sin \theta \frac{\partial \bar{p}_{f}}{\partial \theta}\right)=2 \cos \theta \frac{\Omega}{\omega} \frac{d \varepsilon}{d \tau} \\
& \times\left[1+\frac{C}{R_{1}}\left(\bar{h}_{T}+\frac{\tan \theta}{2} \frac{\partial \bar{h}_{T}}{\partial \theta}\right)+\frac{\sigma}{R_{1}}\left(\phi_{s}+\frac{\tan \theta}{2} \frac{\partial \phi_{s}}{\partial \theta}\right)\right] .
\end{aligned}
$$

Therein, $\bar{h}_{T}$ is the local clearance, and both $\phi$ and $\phi_{\mathrm{s}}$ are the roughness parameters. The boundary condition of fluid pressure $\bar{p}_{f}(\theta, \tau)$ is $\bar{p}_{f}\left(\theta_{r}, \tau\right)=\bar{p}_{r}(\tau)$ and $\bar{p}_{f}\left(\theta_{e}, \tau\right)=0$.

The nominal clearance $\bar{h}$ is given as

$$
\bar{h}=1+\varepsilon \cos \theta .
$$

The total load-carrying capacity is given by summing the load-carrying capacity based 
on the asperities in contact $\bar{W}_{a}$ and the capacity based on the fluid $\bar{W}_{f}$.

$$
\bar{W}_{a}+\bar{W}_{f}=-2 \pi \int_{0}^{\theta e}\left(\bar{p}_{a}+\bar{p}_{f}\right) \cos \theta \sin \theta d \theta
$$

Therein, $\bar{p}_{a}=0$ and $\bar{p}_{f}=\bar{p}_{r}$ in the recess $\left(0<\theta<\theta_{\mathrm{r}}\right)$.

With consideration of fluid compressibility and displacement in the recess, the recess pressure $\bar{p}_{r}(\tau)$ is given as

$$
\bar{V}_{r} \frac{\Omega}{\omega} \frac{d \bar{p}_{r}}{d \tau}=\bar{Q}_{r}-\bar{Q}\left(\theta_{r}\right)-\pi \sin ^{2} \theta_{r} \frac{\Omega}{\omega} \frac{C}{R_{1}} \frac{d \varepsilon}{d \tau} .
$$

Therein, the flow rate $\bar{Q}(\theta)\left(\theta_{\mathrm{r}} \leq \theta \leq \theta_{\mathrm{e}}\right)$ through the land and the flow rate through the capillary restrictor $\bar{Q}_{r}$ are

$$
\begin{aligned}
& \bar{Q}(\theta)=\pi\left(\frac{C}{R_{1}}\right) \sin \theta\left[-\phi \bar{h}^{3} \frac{\partial \bar{p}_{f}}{\partial \theta}+\left(\frac{C}{R_{1}} \bar{h}_{T}+\frac{\sigma}{R_{1}} \phi_{s}\right) \sin \theta \frac{\Omega}{\omega} \frac{d \varepsilon}{d \tau}\right] \text { and } \\
& \bar{Q}_{r}=\pi\left(\frac{C}{R_{1}}\right)\left(\frac{\bar{p}_{s}-\bar{p}_{r}}{\beta}\right) .
\end{aligned}
$$

Therein, $\beta$ is the capillary restrictor parameter $=4 C^{3} l_{\mathrm{c}} /\left(3 r_{\mathrm{c}}{ }^{4}\right), l_{\mathrm{c}}$ is the capillary restrictor length, and $r_{\mathrm{c}}$ is the restrictor radius (See previous papers ${ }^{(11-13)}$ for a case with an orifice restrictor).

The frictional torque $\bar{T}\left(=\bar{T}_{a}+\bar{T}_{f}\right)$ is calculated by summing the torque caused by the asperity $\bar{T}_{a}$, and that of the fluid $\bar{T}_{f}$ given as

$$
\begin{aligned}
& \bar{T}_{a}=\iint_{\bar{A}_{0}} \sin \theta\left\{\xi \bar{\tau}_{p}\left[\left(\frac{\bar{\tau}_{e}}{\bar{\tau}_{p}}\right) d \bar{A}_{e}+d \bar{A}_{p}\right]+(1-\xi) \bar{\tau}_{a d} d \bar{A}_{r}\right\} \text { and } \\
& \bar{T}_{f}=\frac{\pi}{3} \frac{C}{R_{1}} \int_{\theta_{r}}^{\theta_{e}} \frac{\sin ^{3} \theta}{\bar{h}}\left[\phi_{f}+\left(1-2 \frac{\sigma_{2}^{2}}{\sigma^{2}}\right) \phi_{f s}\right] d \theta .
\end{aligned}
$$

Therein, $\bar{A}_{r}$ is the real contact area $=A_{r} / R_{1}{ }^{2}, \bar{A}_{0}$ is the apparent contact area, $\xi$ is the breakdown ratio of an adsorbed film, $\bar{\tau}$ is shear stress $=\tau /\left(p_{s 0} S o\right)$, and $\phi_{f}$ and $\phi_{f s}$ are the roughness parameters after Patir and Cheng; subscript $a d$ is the adsorbed film.

The mean power loss $\bar{L}_{m}$ is defined by summing and averaging the power loss $\bar{L}_{Q m}$ attributable to the leakage flow rate, $\bar{Q}_{o u t}=\bar{Q}\left(\theta_{e}\right)$ and the loss $\bar{L}_{T m}$ of frictional torque:

$$
\bar{L}_{m}=\frac{1}{2 \pi} \int_{0}^{2 \pi}\left(\bar{p}_{s} \bar{Q}_{\text {out }}+\bar{T}\right) d \tau=\bar{L}_{Q_{m}}+\bar{L}_{T m} .
$$

The equation of motion of the sphere along the $z$-axis $\left[\bar{g}=g /\left(\Omega^{2} C\right)\right]$ is

$$
\left(\frac{\Omega}{\omega}\right)^{2} \bar{m} \frac{d^{2} \varepsilon}{d \tau^{2}}=\bar{W}+\bar{W}_{a}+\bar{W}_{f}-\left(\frac{\Omega}{\omega}\right)^{2} \bar{g} \bar{m} .
$$

Under the condition of the plain spherical arc seat in full film lubrication, Eqs. (3), (5), (7) and (10) can be solved analytically; the solutions are shown in the Appendix ${ }^{(11,12)}$. 


\section{Numerical parameters}

Equation (3) is discretized using the finite difference method; Eqs. (6) and (12) are solved using the Runge-Kutta method. The mesh of time $\tau$ in one cycle is divided uniformly by $10^{5}-10^{7}$. The calculation is performed until the relative errors of $\varepsilon, \mathrm{d} \varepsilon / \mathrm{d} \tau$ and $\bar{p}_{r}$ at $\tau=0$ and $2 \pi$ become less than $10^{-3}$, which indicates that the appropriate initial conditions of $\varepsilon$, $\mathrm{d} \varepsilon / \mathrm{d} \tau$ and $\bar{p}_{r}$ at $\tau=0$ and $2 \pi$, and the continuity of the parameters, are satisfied. In addition, the solutions are confirmed by calculating further once with the half mesh in terms of $\tau$.

The representative numerical parameters are the following: $C / R_{1}=10^{-3}, \bar{m}=3.76 \times 10^{-5}$, $R_{\mathrm{w}} / R_{1}=1, R_{1}=10 \mathrm{~mm}, S o=5$ (corresponding to $p_{\mathrm{s}}=10 \mathrm{MPa}$ and $\mu=27.2 \mathrm{mPa} \cdot \mathrm{s}$ ), $\bar{V}_{r}=0.05$, $\beta=3.29 \times 10^{-3}$ (corresponding to $r_{\mathrm{c}}=0.3 \mathrm{~mm}$ ), $\Delta \tau=0 \mathrm{rad}, \theta_{\mathrm{e}}=2 \pi / 3 \mathrm{rad}, \theta_{\mathrm{r}}=\pi / 6, \sigma / \mathrm{C}=0.1$ and $\Omega / \omega=1$. These parameters are fundamentally specified by reference to geometry and conditions of hydraulic equipment.

\section{Results and discussion}

Figures 4-6 show the motion of the sphere and the changes in bearing characteristics for both caulked-socket-type $\left(\theta_{\mathrm{e}}=2 \pi / 3\right)$ and an open (non-caulked)-socket-type $\left(\theta_{\mathrm{e}}=\pi / 2\right)$ bearings. In Fig. 4, the eccentricity ratio $\varepsilon$ is small in the high-pressure period and large in the low-pressure period for both types, whereas the ratio $\varepsilon$ of the open-type socket is markedly large in the low-pressure period. The changes in the recess pressure ratio $p_{\mathrm{r}} / p_{\mathrm{s} 0}$ are similar.

In Fig. 5, the surfaces contact in the latter half time of the high-pressure period. Referring to the bearing's schematic in Fig. 2, it is apparent that, for the caulked-type bearing, in the high-pressure period, the surfaces contact on the lower side of the socket, i.e., the recess edge $\left(\theta=\theta_{\mathrm{r}}\right)$, the surfaces contact on the upper side of the socket, i.e. the caulked socket edge $\left(\theta=\theta_{\mathrm{e}}\right)$. At the transition of the pressures from high to low-pressure periods, for both types of the bearings, cavitation in the bearing socket is apt to occur $\left(A_{\mathrm{cav}} / A_{0}>0\right)$ because the fluid pressure falls substantially as a result of the negative squeeze film action.

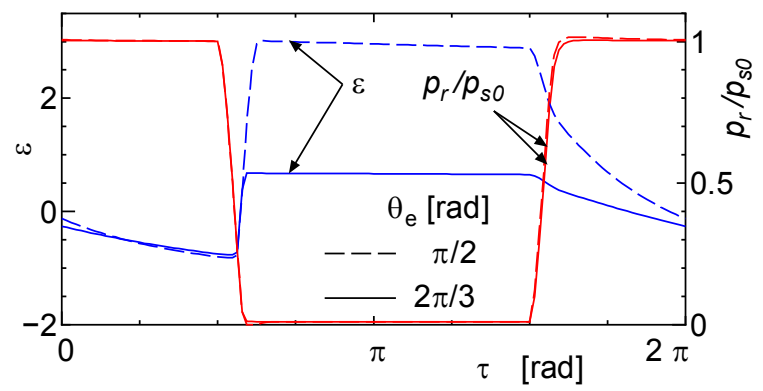

Fig. 4 Effect of caulking on changes in eccentricity ratio $\varepsilon$ and recess pressure ratio $p_{\mathrm{r}} / p_{\mathrm{s} 0}\left(\Delta \tau=0 \mathrm{rad}, \theta_{\mathrm{r}}=\pi / 6\right.$, $\left.\Omega / \omega=1, R_{1} / R_{\mathrm{w}}=1\right)$

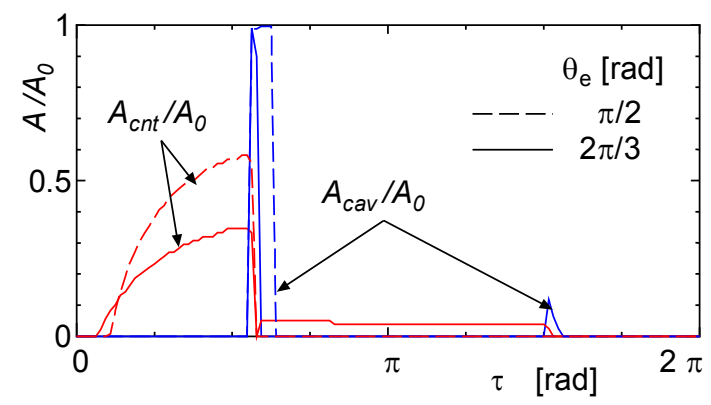

Fig. 5 Effect of caulking on changes in ratios of contact area $A_{\text {cnt }} / A_{0}$ and cavitation area $A_{\text {cav }} / A_{0}(\Delta \tau=0 \mathrm{rad}$, $\left.\theta_{\mathrm{r}}=\pi / 6, \Omega / \omega=1, R_{1} / R_{\mathrm{w}}=1\right)$ 
In Fig. 6, the maximum pressure $p_{\mathrm{a} \max } / p_{\mathrm{s} 0}$ in contact and the leakage flow rate $\bar{Q}_{\text {out }}$ of the caulked-socket-type bearing are shown as markedly smaller than those of the open-socket-type bearing. In particular, the increases in $p_{\mathrm{a} \max } / p_{\mathrm{s} 0}$ before the transition from high-pressure to low-pressure periods and $\bar{Q}_{\text {out }}$ after the transitions are reduced because the motion of the sphere is suppressed by caulking; then the squeeze film action is lessened.

Figures 7 and 8 respectively display the influence of the end angle $\theta_{\mathrm{e}}$ and the recess angle $\theta_{\mathrm{r}}$ of the bearing socket on the difference $\Delta \varepsilon$ in the eccentricity ratios of the maximum $\varepsilon_{\max }$ and minimum $\varepsilon_{\min }$ and the mean power loss $\bar{L}_{m}$, adding the maximum pressure $\bar{p}_{a \max }{ }^{*}$ in contact during the cycle and the mean leakage flow rate $\bar{Q}_{\text {out } m}$. As the end angle $\theta_{\mathrm{e}}$ decreases, the fluctuation $\Delta \varepsilon$ increases; in other words, the bearing stiffness decreases (Fig. 7). In this case, the loss $\bar{L}_{m}$ minimizes close to $\theta_{\mathrm{e}}=\pi / 2$. In contrast, as the recess angle $\theta_{\mathrm{r}}$ decreases, both fluctuation $\Delta \varepsilon$ and the loss $\bar{L}_{m}$ decrease (Fig. 8). The reason of the smaller loss $\bar{L}_{m}$ at the small angle $\theta_{\mathrm{r}}$ is that the solid contact friction based on the pressure $\bar{p}_{a \max }{ }^{*}$ is more effective than the leakage $\bar{Q}_{\text {out } m}$. A small recess angle $\theta_{\mathrm{r}}$ would thus be preferable from the viewpoints of lower power loss and higher bearing stiffness.

Figure 9 shows the effect of the cyclically changing speed of the supply pressure and load fluctuation, corresponding to a rotational speed of a cylinder-block of axial piston pumps, on the maximum $\varepsilon_{\max }$ and minimum $\varepsilon_{\min }$ of the eccentricity ratios of the sphere and mean power loss $\bar{L}_{m}$. The speed of the change is given as the representative angular velocity $\Omega / \omega$, where the angular velocity $\omega$ of the sphere is maintained as constant. As the velocity $\Omega / \omega$ decreases, i.e., at lower speed operation, the ratios $\varepsilon_{\min }$ and $\varepsilon_{\max }$ decrease, so that the surfaces begin to contact close to the recess edge, engendering greater solid friction in mixed lubrication and consequently greater power loss.

For bearing and seal parts used in hydraulic pumps and motors, the load is fundamentally proportional to the discharge pressure. For the present spherical bearing model, the change in the load synchronizes with the change in the supply pressure. However, because of friction between the mating moving parts in the pumps and motors, e.g., frictional parts between pistons and cylinder bores as well as compressibility of liquids, the load change slightly proceeds or delays the pressure change occasionally.

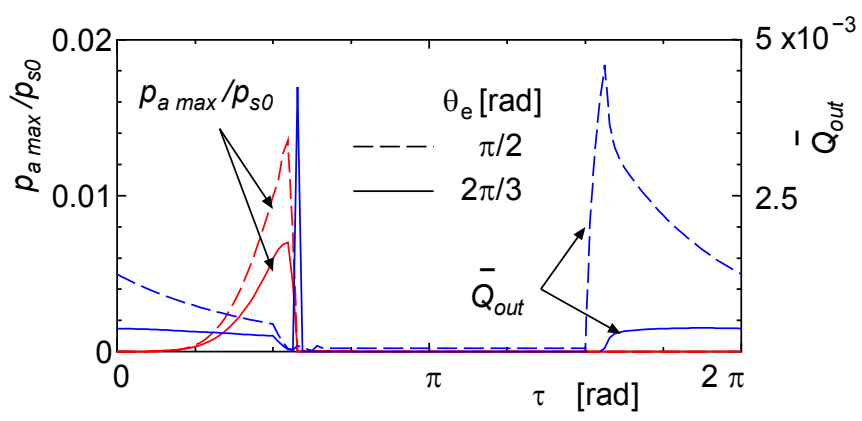

Fig. 6 Effect of caulking on changes in maximum pressure $p_{\mathrm{a} \max } / p_{\mathrm{s} 0}$ in contact and leakage flow rate $\bar{Q}_{\text {out }}$ $\left(\Delta \tau=0 \mathrm{rad}, \theta_{\mathrm{r}}=\pi / 6, \Omega / \omega=1, R_{1} / R_{\mathrm{w}}=1\right)$

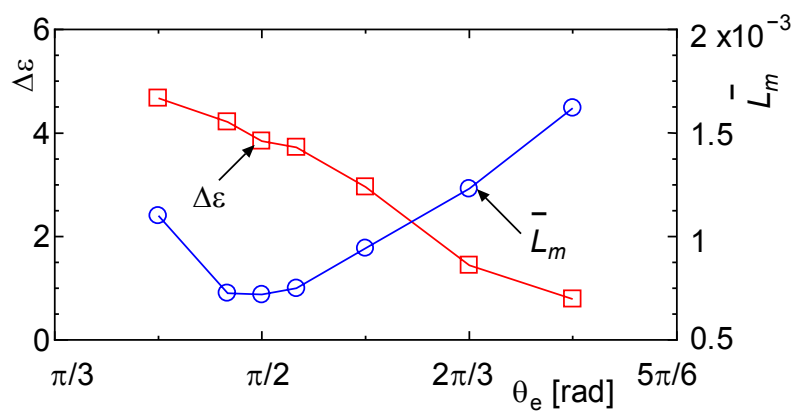

Fig. 7 Effect of end angle $\theta_{\mathrm{e}}$ of bearing socket on eccentricity ratio $\Delta \varepsilon$ fluctuation and mean power loss $\bar{L}_{m}$ $\left(\Delta \tau=0 \mathrm{rad}, \theta_{\mathrm{r}}=\pi / 6 \mathrm{rad}, \Omega / \omega=1, R_{\mathrm{l}} / R_{\mathrm{w}}=1\right)$ 
Figure 10 presents the effect of the time-lag $\Delta \tau$ of the load on the maximum and minimum eccentricity ratios, $\varepsilon_{\max }$ and $\varepsilon_{\min }$, of the sphere. In calculation, the time-dependent input data of the load $W / W_{0}$ is shifted with the lag $\Delta \tau$ against the change in the supply pressure $p_{\mathrm{s}} / p_{\mathrm{s} 0}$ as Fig. 3: When the time-lag $\Delta \tau$ is negative, the load advances the pressure. Under the condition of $\Delta \tau<0$, the influence of $\Delta \tau$ is greater. As the absolute values of $|\Delta \tau|$ increase, the ratio $\varepsilon_{\max }$ becomes noticeably large. The caulked socket enables suppression of the large sphere motion for the wide range of $\Delta \tau$, which contributes to stabilizing the bearing behavior.

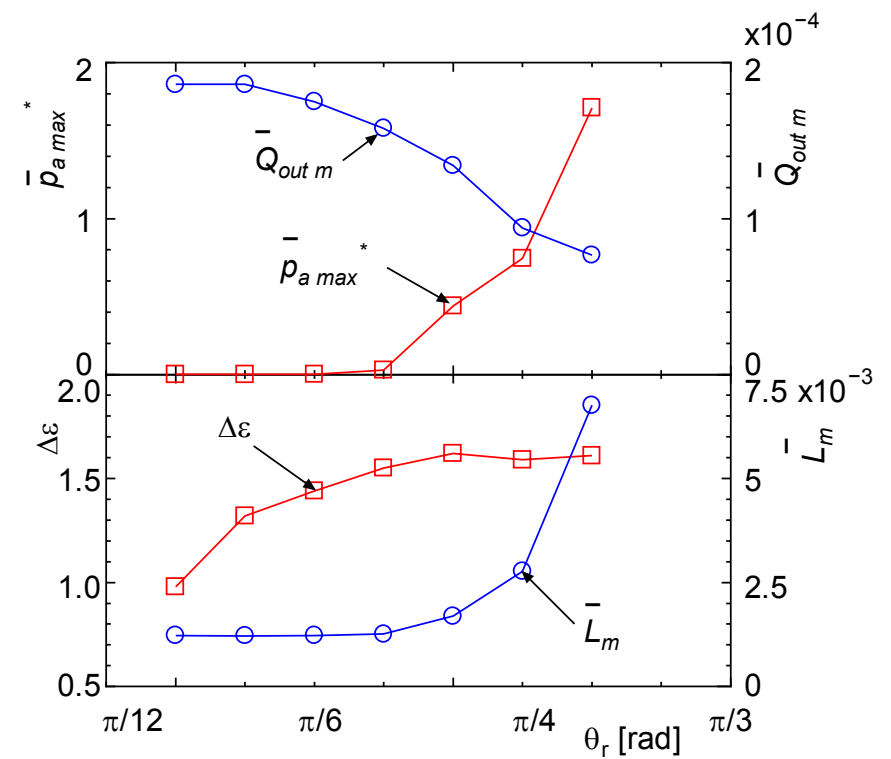

Fig. 8 Effect of recess angle $\theta_{\mathrm{r}}$ of bearing socket on eccentricity ratio $\Delta \varepsilon$ fluctuation, mean power loss $\bar{L}_{m}$, maximum pressure $\bar{p}_{\text {a } \max }{ }^{*}$ in contact and mean leakage flow rate $\bar{Q}_{\text {out } m}\left(\Delta \tau=0 \mathrm{rad}, \theta_{\mathrm{e}}=2 \pi / 3 \mathrm{rad}, \Omega / \omega=1\right.$, $\left.R_{1} / R_{\mathrm{w}}=1\right)$

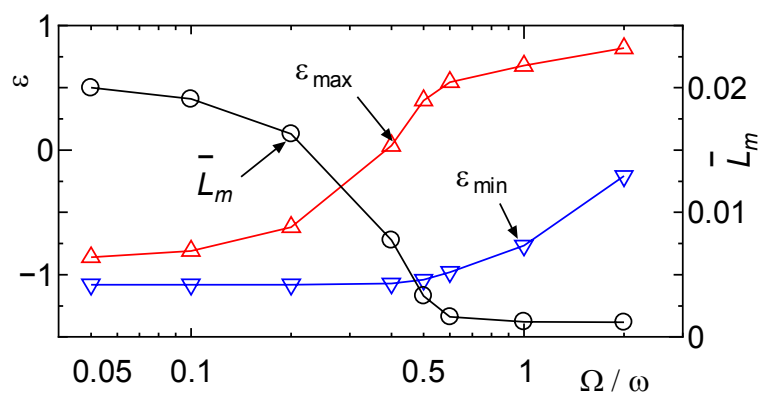

Fig. 9 Effect of angular velocity $\Omega / \omega$ on maximum and minimum eccentricity ratios $\varepsilon_{\max }$ and $\varepsilon_{\min }$ of the sphere and mean power loss $\bar{L}_{m}\left(\Delta \tau=0 \mathrm{rad}, \theta_{\mathrm{e}}=2 \pi / 3 \mathrm{rad}, \theta_{\mathrm{r}}=\pi / 6 \mathrm{rad}, R_{1} / R_{\mathrm{w}}=1\right)$

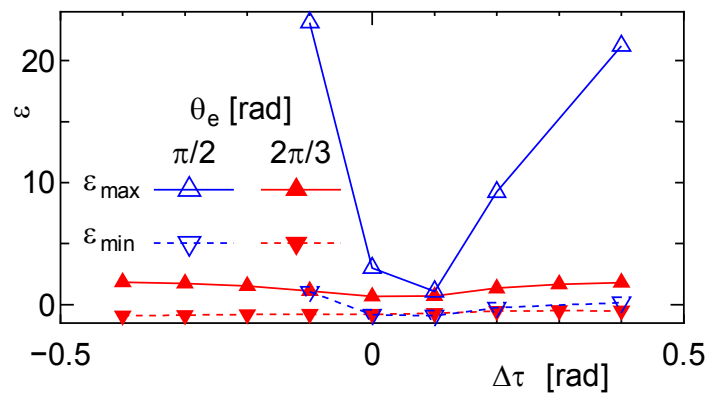

Fig. 10 Effect of time-lag $\Delta \tau$ and caulking on maximum and minimum eccentricity ratios $\varepsilon_{\max }$ and $\varepsilon_{\min }$ of the sphere $\left(\theta_{\mathrm{r}}=\pi / 6 \mathrm{rad}, \Omega / \omega=1, R_{1} / R_{\mathrm{w}}=1\right)$ 
Figure 11 depicts the effect of the sphere radius $R_{1} / R_{\mathrm{w}}$ on the mean power loss $L_{\mathrm{m}} / L_{\mathrm{m} 0}$ and dynamic stiffness $\lambda / \lambda_{0}$, where $\lambda$ is defined as $\lambda=\left(|W|_{\max }-|W|_{\min }\right) /\left(\varepsilon_{\max }-\varepsilon_{\min }\right)$, the equivalent radius $R_{\mathrm{w}}$ of the load is kept constant, and the loss and the stiffness are normalized by the power loss $L_{\mathrm{m} 0}$ and the stiffness $\lambda_{0}$ of the caulked-socket-type bearing at $R_{1} / R_{\mathrm{w}}=1$. It is readily apparent that the optimum sphere radius that gives the power loss being minimized exists close to the condition of $R_{1} / R_{\mathrm{w}} \cong 1$ for both socket-type bearings. Smaller loss $L_{\mathrm{m}} / L_{\mathrm{m} 0}$ is given with the caulked-socket-type bearing in a wide range of $R_{1} / R_{\mathrm{w}}$, whereas the minimum of $L_{\mathrm{m}} / L_{\mathrm{m} 0}$ of the open-socket-type bearing is slightly smaller than that of $L_{\mathrm{m}} / L_{\mathrm{m} 0}$ of the open-socket-type.

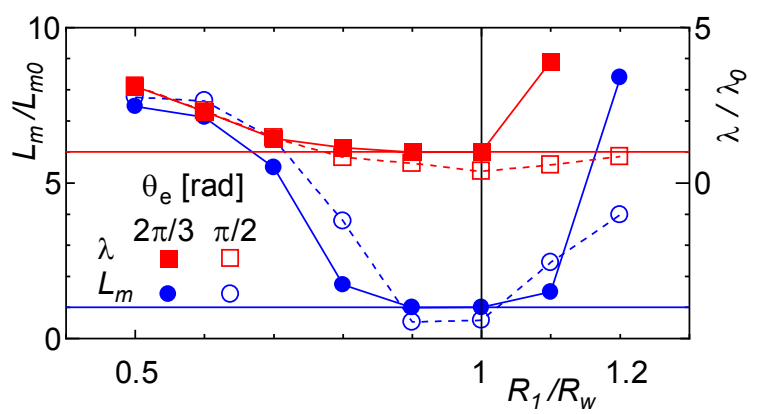

Fig. 11 Effect of sphere radius $R_{1} / R_{\mathrm{w}}$ on normalized mean power loss $L_{\mathrm{m}} / L_{\mathrm{m} 0}$ and dynamic stiffness $\lambda / \lambda_{0}$

$$
\left(\Delta \tau=0 \mathrm{rad}, \theta_{\mathrm{r}}=\pi / 6 \mathrm{rad}, \Omega / \omega=1\right)
$$

\section{Concluding remarks}

To overcome the discrepancy in the bearing's operation between the conventional theory's assumption based on fluid film lubrication and a practical operating condition enforced in mixed lubrication, hydrostatic ball joints of swash-plate-type axial piston pumps and motors are modeled as hydrostatic spherical bearings including caulking of the bearing socket, cyclic change in the load, roughness interaction, and asperity contact. Subsequently, a series of the governing equations are derived and the computer code is developed. The tribological behavior of the caulked-socket-type and open-socket-type hydrostatic spherical bearings is simulated in mixed and fluid film lubrication under a non-steady-state condition. The numerical simulation reveals the following.

The sphere's eccentricity increases in the low supply pressure period. As the time-lag of the load increases, the sphere jumps greatly, especially for the open-type socket bearing. The caulked socket enables suppression of sphere jumping and maintains the bearing stability: the bearing performance is improved by caulking.

The pressure in contact is large at the termination of the high-pressure period and the leakage flow rate is large at the commencement of the high-pressure period. Under the low-speed conditions, both the maximum and minimum of the eccentricity ratios decrease and the power losses increase.

As the recess angle decreases, the power loss decreases and the bearing stiffness increases. Within the calculation parameters, the power loss is minimized in the condition where the socket radius of the bearing almost equals the equivalent radius of the load, which gives an optimal dimension of the piston assembly.

\section{Acknowledgements}

The author expresses his sincere appreciation to Professor Emeritus Atsushi Yamaguchi of Yokohama National University and to Professor Emeritus Mitsuru Fujiwara of Muroran Institute of Technology for their thoughtful encouragement. 


\section{Appendix}

For a plane hydrostatic spherical bearing with a rigid body in fluid film lubrication the Reynolds equation is given as:

$$
\frac{1}{\sin \theta} \frac{\partial}{\partial \theta}\left(\bar{h}^{3} \sin \theta \frac{\partial \bar{p}}{\partial \theta}\right)=2 \cos \theta \frac{\Omega}{\omega} \frac{d \varepsilon}{d \tau}
$$

Therein, the clearance is given as $\bar{h}=\varepsilon \cos \theta$ for the fitted-type bearing and $\bar{h}=1+\varepsilon \cos \theta$ for the clearance-type bearing. Solving Eq. (A1) with $x=\cos \theta$, one obtains

$$
\bar{p}(x)=\frac{G_{e}-G(x)}{\Delta G} \cdot \bar{p}_{r}+\frac{\Omega}{\omega} \frac{\Delta G_{1} G(x)-\Delta G G_{1}(x)+G_{1 r} G_{e}-G_{1 e} G_{r}}{\varepsilon^{3} \Delta G} \frac{d \varepsilon}{d \tau} .
$$

Assuming that cavitation is negligible, Eq.(A2) is integrated in terms of $x$ over $x=x_{\mathrm{e}} \sim x_{\mathrm{r}}$, the load-carrying capacity $\bar{W}_{f}$ is given as

$$
\bar{W}_{f}=\pi \frac{\Delta I}{\Delta G} \bar{p}_{r}-\pi \frac{\Omega}{\omega} \frac{\Delta G_{2} \Delta I-\Delta G \Delta I_{1}}{\varepsilon^{3} \Delta G} \frac{d \varepsilon}{d \tau} .
$$

Putting $X=\varepsilon x$ (fitted-type bearing) or $X=1+\varepsilon x$ (clearance-type bearing), the leakage flow rate $\bar{Q}(x)$ is

$$
\bar{Q}(x)=\frac{\pi X\left(1-x^{2}\right)}{R_{1} / C}\left(X^{2} \frac{\partial \bar{p}}{\partial x}+\frac{C}{R_{1}} \frac{\Omega}{\omega} \frac{d \varepsilon}{d \tau}\right) .
$$

The frictional torque $\bar{T}_{f}$ is

$$
\bar{T}_{f}=\frac{\pi}{3} \frac{C}{R_{1}} \frac{\Delta J}{\varepsilon} .
$$

The parameters $G, I$ and $J$ are defined below. For clearance-type bearings, the equations are the same by putting $\varepsilon=1$ in Eqs. (A2), (A3) and (A5). It is noteworthy that the fitted-type bearing is invalid for rough surfaces $(\sigma>0)$ and caulked sockets $\left(\theta_{\mathrm{e}} \geq \pi / 2\right)$.

The parameters $\Delta G=G\left(x_{\mathrm{e}}\right)-G\left(x_{\mathrm{r}}\right), \Delta I=I\left(x_{\mathrm{e}}\right)-I\left(x_{\mathrm{r}}\right), \Delta J=J\left(x_{\mathrm{e}}\right)-J\left(x_{\mathrm{r}}\right), \Delta G_{1}=G_{1}\left(x_{\mathrm{e}}\right)-G_{1}\left(x_{\mathrm{r}}\right)$, $\Delta G_{2}=G_{2}\left(x_{\mathrm{e}}\right)-G_{2}\left(x_{\mathrm{r}}\right)$ and $\Delta I_{1}=I_{1}\left(x_{\mathrm{e}}\right)-I_{1}\left(x_{\mathrm{r}}\right)$ are given as follows.

a) For fitted-type bearings

$$
\begin{aligned}
& G(x)=1 /\left(2 x^{2}\right)+\log \left(1 / x^{2}-1\right) / 2 \\
& I(x)=1 /\left(2 x^{2}\right) \\
& J(x)=x^{2} / 2-\log x \\
& G_{1}(x)=\log \left(1 / x^{2}-1\right) / 2 \\
& G_{2}(x)=-1 /\left(2 x^{2}\right) \\
& I_{1}(x)=-\log x-1 /\left(2 x^{2}\right)
\end{aligned}
$$

b) For clearance-type bearings (superscript represents $\varepsilon$; no superscript means $\varepsilon \neq 0, \pm 1$ ) 


$$
\begin{aligned}
& G(x)=\frac{-\varepsilon\left(5+4 \varepsilon x-\varepsilon^{2}\right)}{2(1+\varepsilon)^{2}(1-\varepsilon)^{2}(1+\varepsilon x)^{2}} \\
&+\frac{\varepsilon\left(3+\varepsilon^{2}\right)}{(1+\varepsilon)^{3}(1-\varepsilon)^{3}} \log (1+\varepsilon x) \\
&-\frac{1}{2}\left[\frac{\log (1+x)}{(1-\varepsilon)^{3}}-\frac{\log (1-x)}{(1+\varepsilon)^{3}}\right] \\
& G_{1}(x)^{0}=-\log [(1+x) /(1-x)] / 2 \\
& G_{1}(x)^{ \pm 1}=\frac{ \pm 10+9 x \pm 3 x^{2}}{24(1 \pm x)^{3}}-\frac{1}{16} \log \left(\frac{1+x}{1-x}\right)
\end{aligned}
$$

$$
\begin{aligned}
& I(x)^{0}=-x \\
& I(x)^{ \pm 1}=( \pm 1+x) /\left[2(1 \pm x)^{3}\right]
\end{aligned}
$$$$
J(x)=\frac{-x(2-\varepsilon x)}{2 \varepsilon^{2}}+\frac{1-\varepsilon^{2} x}{\varepsilon^{3}} \log (1+\varepsilon x)
$$$$
J(x)^{0}=x^{3} / 3-x
$$$$
J(x)^{ \pm 1}=J(x)
$$

$$
\begin{aligned}
G_{1}(x) & =\frac{-1-3 \varepsilon^{2}-4 \varepsilon^{3} x}{2 \varepsilon(1+\varepsilon)^{2}(1-\varepsilon)^{2}(1+\varepsilon x)^{2}} \\
& +\frac{\varepsilon\left(3+\varepsilon^{2}\right)}{(1+\varepsilon)^{3}(1-\varepsilon)^{3}} \log (1+\varepsilon x) \\
& -\frac{1}{2}\left[\frac{\log (1+x)}{(1-\varepsilon)^{3}}-\frac{\log (1-x)}{(1+\varepsilon)^{3}}\right]
\end{aligned}
$$

$G_{1}(x)^{0}=x-\log [(1+x) /(1-x)] / 2$

$$
G_{1}(x)^{ \pm 1}=\frac{\mp 2-3 x \pm 3 x^{2}}{24(1 \pm x)^{3}}-\frac{1}{16} \log \left(\frac{1+x}{1-x}\right)
$$

$$
\begin{aligned}
& G_{2}(x)=-1 /\left[2 \varepsilon(1+\varepsilon x)^{2}\right] \\
& G_{2}(x)^{0}=x \\
& G_{2}(x)^{ \pm 1}=\mp 1 /\left[2(1 \pm x)^{2}\right]
\end{aligned}
$$

$$
\begin{aligned}
& I_{1}(x)=-1 /\left[\varepsilon^{3}(1+\varepsilon x)\right]-\left(1-x^{2}\right) /\left[2 \varepsilon(1+\varepsilon x)^{2}\right]-\log (1+\varepsilon x) / \varepsilon^{3} \\
& I_{1}(x)^{0}=x-x^{3} / 3 \\
& I_{1}(x)^{ \pm 1}=\mp(3 \pm x) /[2(1 \pm x)] \pm \log (1 \pm x)
\end{aligned}
$$




\section{References}

(1) Yamaguchi, A., Performance of Spherical Bearings for Piston Pumps and Motors (in Japanese), J. Japan Hydraulics and Pneumatics Society, Vol.1, No.1 (1970), pp.10-18.

(2) Iboshi, N. and Yamaguchi, A., Characteristics of a Slipper Bearing for Swash Plate Type Axial Piston Pumps and Motors (1st Report, Theoretical Analysis). Bull. Japan Society Mechanical Engineers, Vol.25, No.210 (1982), pp.1921-1930.

(3) Sasaki, T., Mori, H. and Hirai, A., Theoretical Study of Hydrostatic Thrust Bearings, Bull. JSME, Vol.2 (1959), pp.75-79.

(4) Dowson, D. and Taylor, C.M., A Re-Examination of Hydrosphere Performance, ASLE Trans., V1.10 (1967), pp.316-324.

(5) Hamrock, B.J., Fundamentals of Fluid Film Lubrication, (1994), pp.293-312, McGraw-Hill.

(6) Japanese Society of Tribologists (ed.), Tribology Handbook (in Japanese), (2001), pp.83-92; 306-308, Yoken-do.

(7) Kazama, T. and Yamaguchi, A., Application of A Mixed Lubrication Model for Hydrostatic Thrust Bearings of Hydraulic Equipment, J. Tribology, Trans. ASME, Vol.115 (1993), pp.686-691.

(8) Hazaki, E. and Imai, M., A Study of Spherical Bearings of Hydraulic Motor (in Japanese). Trans. Japan Society Mechanical Engineers, C, Vol.49, No.442 (1983), pp.1022-1028.

(9) Fang, Y. and Ikeya, M., Friction Characteristics of the Ball Joint in a Swashplate Type Axial Piston Motor (in Japanese). J. Japan Hydraulics and Pneumatics Society, Vol.23, No.2 (1992), pp.189-194.

(10) Yacout, A.W., Ismaeel, A.S. and Kassab, S.Z., The Combined Effects of the Centripetal Inertia and the Surface Roughness on the Hydrostatic Thrust Spherical Bearings Performance, Tribology International, Vol.40, No.3 (2007), pp.522-532.

(11) Kazama, T., Optimum Design on Hydrostatic Spherical Bearings (Analytical Solutions in Fluid Film Lubrication) (in Japanese), Trans. JSME, C, 64-619 (1998), 1049-1054.

(12) Kazama, T., Mixed Lubrication Characteristics of Hydrostatic Spherical Bearings for Piston Pumps and Motors (in Japanese), Trans. JSME, C, 67-658 (2001), 2014-2018.

(13) Kazama, T., Mixed Lubrication Characteristics of Dynamically Loaded Hydrostatic Spherical Bearings (in Japanese), Trans. JSME, C, 69-684 (2003), 2200-2205.

(14) Yamaguchi, A. and Matsuoka, H., A Mixed Lubrication Model Applicable to Bearing/Seal Parts of Hydraulic Equipment, J. Tribology, Trans. ASME, Vol.114 (1992), pp.116-121.

(15) Greenwood, J.A. and Williamson, J.B.P., Contact of Nominally Flat Surfaces, Proc. Roy. Soc., London, Ser. A, Vol.295 (1966), pp.300-319.

(16) Patir, N. and Cheng, H.S., Application of Average Flow Model to Lubrication Between Rough Sliding Surfaces, J. Lubrication Technology, Trans. ASME, Vol.101 (1979), pp.220-230. 